\section{Author reply: Bortezomib and restoration of chemosensitivity}

\author{
Chor Sang Chim, Yu Yan Hwang, Clara Pang and Tony W. Shek
}

We thank Dr Gozzetti for his comments about a possible delayed clinical response to bortezomib instead of chemosensitivity restoration by bortezomib because the response to bortezomib may sometimes be delayed. Moreover, documentation of multi-drug resistance (MDR) would have clarified this postulation before and after Vel-CMP therapy.

We would like to explain the observed response, including tumor response and paraprotein response, in terms of the sequence of events to different sequential chemotherapy regimens as listed in Table 1. Interestingly, our patient relapsed with plasmacytomas in the pancreas and pleura without emergence of IgA paraprotein, which only appeared during further disease progression (Table 1). Of the major chemotherapy regimens, three contained bortezomib including Vel-Dex, PAD and Vel-CMP. Therefore, our patient actually had tumor progression (at the head of the pancreas) after the first bortezomib-based regimen Vel-Dex. Moreover, subsequent treatment with the second bortezomib-
Table 1 | Sequential chemotherapy regimens in view of no response or progressive disease

\begin{tabular}{llllll}
\hline \multirow{2}{*}{ Response parameters } & \multicolumn{4}{c}{ Drug regimen } \\
\cline { 2 - 5 } & CEOP & Vel-Dex* & MP and RT & PAD* & Vel-CMP* \\
\hline Tumor & No response & Progression & No response & No response & $\begin{array}{l}\text { Complete } \\
\text { remission }\end{array}$ \\
\hline IgA paraprotein & Absent & Absent & Progressed & Further & $\begin{array}{l}\text { Complete } \\
\text { remission }\end{array}$ \\
\hline $\begin{array}{l}\text { *Regimens that contained bortezomib. Abbreviations: CEOP, cyclophosphamide, epirubicin, vincristine and prednisolone; } \\
\text { MP, melphalan and prednisolone; PAD, bortezomib, adriamycin and dexamethasone; RT, radiotherapy; Vel-CMP, bortezomib, } \\
\text { cyclophosphamide, melphalan and prednisolone; Vel-Dex, bortezomib and dexamethasone. }\end{array}$
\end{tabular}

based regimen, $\mathrm{PAD}$, did not cause a response in the pancreatic tumor but a further rise of IgA paraprotein. Our patient actually had progressive disease on both occasions of exposure to bortezomib. Therefore, while Dr Gozzetti is entirely correct that response to bortezomib may be as late as after eight cycles, our patient's complete response (both tumor and IgA paraprotein) would be less likely to be due to bortezomib only.

We agree that documentation of MDR would provide evidence that the myeloma plasma cells were resistant before Vel-CMP. However, this was not possible as we did not have RNA from CD138selected plasma cells before Vel-CMP. Furthermore, MDR study is also not possible after Vel-CMP as our patient had gone into complete remission, when the marrow was devoid of malignant plasma cells. This would also have precluded the study of MDR after completion of Vel-CMP.

Queen Mary Hospital, Hong Kong

Correspondence:C. S. Chim, Department of Medicine, Queen Mary Hospital, Pokfulam Road, Hong Kong jcschim@hku.hk

doi: $10.1038 /$ nrclinonc.2009.15-c2 